\title{
NSUN6, a Gene Related With m5c Methylation, May Serve as a Biomarker for Pan-cancer
}

Baishun Ma ( $\sim 2239850726 @ q q . c o m$ )

Heze Municipal Hospital https://orcid.org/0000-0002-4399-7555

Pu Wang

Heze Municipal Hospital

\section{Research Article}

Keywords: m5C, NSUN6, TCGA, pan-cancer analysis

Posted Date: March 1st, 2022

DOI: https://doi.org/10.21203/rs.3.rs-1352812/v1

License: (c) (i) This work is licensed under a Creative Commons Attribution 4.0 International License. Read Full License 


\section{Abstract}

Background: 5-methylcytosine ( $\mathrm{m} 5 \mathrm{c}$ ) is a common chemical alteration in DNA and RNA, however unlike DNA modifying enzymes, the methyl-transferases that make $\mathrm{m} 5 \mathrm{c}$ changes in RNA are little understood. The putative RNA methyltransferase NSUN6 is a member of the Nol1/Nop2/SUN domain (NSUN) protein family, but its function in most malignancies is uncertain.

Methods: The relevance of NSUN6 in tumor metabolism and tumor immunity was investigated in the present study. Through a pan-cancer analysis using multiple databases. The expression levels, prognosis, tumor progression, immunological checkpoints, and immune metabolism of NSUN6 in 33 cancers were determined using the Cancer Genome Atlas (TCGA), Genotype-Tissue Expression (GTEx), and Cancer Cell Line Encyclopedia (CCLE) datasets. The current study looked at pathways, immunological checkpoints, immune neo-antigens, immune microenvironment, tumor mutational burden (TMB), microsatellite instability (MSI), DNA mismatch repair (MMR), and the usefulness of NSUN6 methyl-transferases. For quantitative analysis and panoramic description, the $\mathrm{R}$ package was employed.

Results: We discovered that NSUN6 is variably expressed in pan carcinomas, and that NSUN6 was typically related with the prognosis of ACC, COAD, KIRC, SARC, and THCA, where low expression of NSUN6 was associated with a significantly worse prognosis. While high NSUN6 expression has been linked to a worse prognosis in patients with BLCA, LGG, and PAAD. Second, we confirmed that the level of NSUN6 expression was linked to tumor immune infiltration and the tumor microenvironment, particularly in BLCA, KIRC, and KIRP. Finally, in addition to TMB and MSI in pan-malignancies, NSUN6 was linked to immunological neo-antigen and immune checkpoint gene expression profiles in all cancers.

Conclusion: NSUN6 is variably expressed in pan-cancers and has the potential to be used as a predictive biomarker for tumors.

\section{Highlights}

(1) The degree of NSUN6 expression differs between tumor types.

(2) While NSUN6 expression was substantially correlated with prognosis in patients with malignancies, increased NSUN6b expression was typically associated with a negative prognosis across multiple datasets.

(3) NSUN6 was found to be highly linked with tumor immunity and to interact with a variety of immune cells in many forms of cancer.

(4) Correlations between NSUN6 expression and ICP, TMB, MSI, MMRs, and DNA methylation were significant.

\section{Introduction}


People's living standards, dietary habits, and living situations change as a result of economic and social growth, and the incidence of cancer and mortality has gradually increased, becoming a global burden on human health $[1,2]$. Traditional treatments for cancer which including surgery, chemotherapy, and radiotherapy are still the most common treatments, and many cancer sufferers do not achieve the five year survival rate that is optimal $[3,4]$. Cancer has become one of the leading causes of mortality in both industrialized and developing countries, putting pressure on humans to create new treatments for all types of tumors [5]. The use of tumor markers in the detection and treatment of cancer has given cancer therapy a fresh lease on life [6]. One of the most essential ways for identifying effective tumor markers is to conduct a pan-cancer investigation [7].

The action of seven members of the NOL1/NOP2/SUN domain (NSUN) family, including NSUN1, NSUN2, NSUN3, NSUN4, NSUN5, NSUN6, and NSUN7, and DNA methyltransferase 2/tRNA methyltransferase 1(DNMT2/TRMT1), is thought to be the mechanism by which $\mathrm{m} 5 \mathrm{C}$ attaches to various RNA molecules [8]. NSUN2 is a nucleolin that is involved in tissue homeostasis, spindle stability, and early embryogenesis [9]. In breast cancer and prostate cancer, knocking down Sphingosine Kinase 1 (SK1) is linked to NSUN2 downregulation [10], suggesting that SK1 of drug inhibitor may be beneficial to over-expression of NSUN2 in cancer [11]. Direct deletion of nsun2 in HeLa cells can improve sensitivity to 5-fluorouracil (5-FU), but not cisplatin or paclitaxel, at the same time [12]. Through the interaction of NELFB and RPS6KB2, the 5mC RNA methyltransferase NSUN6 regulates the response of glioblastoma to temozolomide (TMZ) [13]. Cox regression analysis showed that the expression of DNMT1, TET2 and NSUN6 was related to the prognosis of Head and neck squamous cell carcinoma (HNSCC)[14]. Although these studies suggest that NSUN6 plays an important role in a variety of tumors, there is no pan-cancer analysis of NSUN6, and the role of NSUN6 in a variety of tumors has not been fully elucidated.

Histological approaches have steadily become the center of study since the emergence of highthroughput sequencing. For cancer research, histological techniques offer a new perspective [15]. Pancancer analysis was used in this work to examine the expression, prognosis, TMB and MSI of NSUN6 in 33 cancers. Apart from that, we looked at the role of NSUN6 in the immune microenvironment and the relationship between immune-related antigens and checkpoint genes. In pan-cancer tissues, we found that NSUN6 gene expression influenced the expression of DNA repair genes and methyltransferase. Using gene set enrichment analysis, we found that NSUN6 regulates the signal pathways related to spermatogenesis, asthma and inflammation.

\section{Methods And Materials}

\section{Data Source}

Studies were based on The Cancer Genome Atlas (TCGA, https://portal.gdc.cancer.gov/) dataset [16], Genotype-Tissue Expression (GTEx, https://gtexportal.org/) dataset [17], Cancer Cell Line Encyclopedia (CCLE, https://portals.broadinstitute. org/) dataset [18] and the TIMER (https://cistrome.shinyapps.io/ timer/) dataset [19]. For the study of NSUN6 expression in 27 cancers, data from the TCGA and GTEx 
databases were used, as were clinical records and gene expression data from tumor and normal samples. Analysis of NSUN6 gene expression in 31 GTEx dataset tumors was also performed. CCLE and TIMER data was downloaded to analyze gene expression differences between cancer cell lines, and RNA sequencing datasets were downloaded for each cell line in the CCLE dataset to examine gene expression differences between cancer cell lines. Only open access data were used in this study, hence ethics committee permission was not required.

\section{Analysis of NSUN6 Expression Levels and Prognosis in Pan- Cancer}

In this study, the R package (edgeR) was used to analyze the differential levels of NSUN6 expression in the dataset[20]. For the expression of NSUN6 in different tumor cells and other normal tissues, we used Kruskal-Wallis test analysis method, and the R package ggplot2 presented the figures[21]. A one-way Cox regression test was utilized to examine the relationship between NSUN6 and patient survival in pancancer prediction analysis. $\mathrm{K}-\mathrm{M}$ test was also used to measure the survival of patients in comparison with others. Forest plot visualization software was used to plot the images.

\section{Gene Set Enrichment Analysis}

This effective tool for assessing gene expression data and studying statistically significant and persistent differences between various groups with various biological states is known as gene set enrichment analysis (GSEA) [22]. The signaling pathway of NSUN6 was analyzed by gene set enrichment analysis. The Kyoto Encyclopedia of Genes and Genomes (KEGG) pathway enrichment analysis was performed with the R package clusterProfiler.[23] A second dataset utilized for GSEA analysis is the Molecular Signature Database of immune cells that control the level of immunological activation by preserving their normal immune function in vivo. Results showed that NSUN6 expression levels were associated with 47 common immune checkpoint gene expression. Multiple malignancies were examined for correlations between NSUN6 expression levels and expression levels of other immune checkpoint genes using the R package (limma, reshape2, RColorBrewer). A scanner was used to count and identify each tumor sample's neoantigens.

\section{Correlation Analysis of NSUN6 in Immune Infiltration and Tumor Microenvironment}

With the use of a purity-adjusted Spearman correlation analysis, we looked at the relationship between the NSUN6 gene and six different immune cell infiltrations to see how well it performed. The tumor microenvironment-related scores of patients, such as estimation score, tumor stromal score, and immune score, were also assessed using an ESTIMATE algorithm using a standardized expression matrix. Statistical significance was defined as a p-value of 0.05 or lower.

\section{Tumor Mutational Burden and Microsatellite Instability Analysis}


The total TMB was calculated using the Perl language and a R program ( $\mathrm{fmsb}$ ) and correlated with NSUN6 expression levels in pan-cancer patients. It is possible to employ MSI to screen for, diagnose, and predict pancreatic cancer because tumors have unique microsatellite alleles relative to normal tissue. The fmsb package in R was used to examine the relationship between MSI and NSUN6 expression levels in pan-cancer.

\section{DNA Mismatch Repair Gene Mutation and DNA Methylation Analysis}

The Spearman correlation approach was used to examine the relationship between MMR gene expression levels and NSUN6 gene expression levels. Gene expression is greatly influenced by DNA methylation, as well. The Spearman correlation approach was used to examine the correlation between DNMT1 (DNMT1), DNMT2 (DNMT2), DNMT3A (DNMT3B), and NSUN6 expression levels.

\section{Results}

\section{NSUN6 expression was significantly upregulated in pan- cancer}

NSUN6's expression in various cancer databases was first evaluated in this work in order to determine the level of NSUN6 expression in pan-cancer. Data from GTEx (Fig. 1A), CCLE (Fig. 1B), and TCGA (Fig. 1C) showed NSUN6 expression levels to be similar across the three datasets. Figure 1A shows that NSUN6 expression was lower in muscle and blood than in other tissues, but higher in the testis and pituitary than in other tissues. NSUN6 expression was lower in salivary glands than in other tissues, as depicted in Fig. 1B. Comparatively, the TCGA database shows that the NSUN6 gene is significantly expressed in COAD (ESCA) and HNSC (KIRC), as well as in LUAD (LUSC), PRAD (RAD), READ (STAD), and KIRP (KIRP). The expression of NSUN6 in the TCGA and GTEx datasets was further integrated in light of the small amount of normal sample data in TCGA. The final analysis revealed that NSUN6 expression was downregulated in CHOL, GBM, KICH, LIHC, OV, SKCM, TGCT, THCA, and UCEC, but elevated in BRCA, COAD, ESCA, HNSC, KIRC, KIRP, LAML, LGG, LUSC, PAAD, PRAD, READ, STAD, and UCS after integration of 27 tumor modules (Fig. 1D).

\section{Prognostic analysis of NSUN6 in pan-cancer}

In the above study, the expression levels of NSUN6 in various tumor tissues were revealed. To understand the relationship between NSUN6 and tumor prognosis, one-way Cox regression analysis was used to analyze NSUN6 expression levels and patient prognosis. Based on the TCGA database data, NSUN6 expressions were grouped into high and low expression groups according to the median value of NSUN6 expression in each tumor. It was further observed that NSUN6 was only expressed in ACC (HR = 1.1, 95Cl\%: 1.02-1.17, $\mathrm{P}=0.0083), \mathrm{BLCA}(\mathrm{HR}=0.97,95 \mathrm{Cl} \%$ : $0.95-0.99, \mathrm{P}=0.0025), \mathrm{COAD}(\mathrm{HR}=1.08,95 \mathrm{Cl} \%$ : 
1.02-1.14, $\mathrm{P}=0.011), \mathrm{KIRC}(\mathrm{HR}=1.07,95 \mathrm{Cl} \%: 1.02-1.13, \mathrm{P}=0.0033), \mathrm{LGG}(\mathrm{HR}=0.94,95 \mathrm{Cl} \%: 0.91-0.97$, $\mathrm{P}=0.00033)$, PAAD (HR = 0.91, 95Cl\%: 0.84-0.98, $\mathrm{P}=0.012), \operatorname{SARC}(\mathrm{HR}=1.07,95 \mathrm{Cl} \%: 1.01-1.12, \mathrm{P}=$ $0.012)$ and $\mathrm{T} \mathrm{HCA}(\mathrm{HR}=1.12,95 \mathrm{Cl} \%: 1.02-1.22, \mathrm{P}=0.014)$ with of prognostic significance in 33 tumors (Fig. 2). In addition, the relationship between NSUN6 and ACC, BLCA, COAD, KIRC, LGG, PAAD, SARC, and THCA was further observed by K-M plotting survival curves (Fig. 3). It suggested that there was an association between NSUN6 and tumor prognosis, especially with LGG. Considering the possible existence of non-tumor-related deaths during follow- up, we analyzed the relationship between NSUN6 expression levels and prognostic DSS (disease-specific survival) in 33 tumors of TCGA. The results of the analysis showed that NSUN6 was related to $A C C(H R=1.1,95 \mathrm{Cl} \%: 1.03-1.18, P=0.0065), B L C A(H R=$ 0.96, 95Cl\%: 0.64-0.99, $\mathrm{P}=0.0042), \mathrm{LGG}(\mathrm{HR}=0.95,95 \mathrm{Cl} \%: 0.91-0.98, \mathrm{P}=0.00088), \mathrm{PAAD}(\mathrm{HR}=0.89$, 95Cl\%: $0.81-0.97, \mathrm{P}=0.0078), \mathrm{PCPG}(\mathrm{HR}=1.24,95 \mathrm{Cl} \%: 1-1.54, \mathrm{P}=0.048), \mathrm{SARC}(\mathrm{HR}=1.07,95 \mathrm{Cl} \%: 1.01-$ $1.13, P=0.018), \operatorname{SKCM}(H R=0.96,95 \mathrm{Cl} \%: 0.92-1, P=0.036)$, and $\mathrm{THCA}(\mathrm{HR}=1.21,95 \mathrm{Cl} \%: 1.09-1.33, \mathrm{P}=$ 0.00017 ) revealing that NSUN6 and DSS had prognostic significance (Fig. 4). NSUN6 was also shown to be a predictive predictor of tumor DSS by the K-M survival curve (Fig. 5).

\section{Tumor metabolic and immune signaling pathways involved in NSUN6}

A GSEA analysis was performed to examine the signaling enrichment of KEGG and markers in both groups according to NSUN6 gene expression, which was separated into high and low groups, in order to better understand the relationship between NSUN6 involvement in cancer metabolism and tumor immunity. In addition, we identified the six signaling pathways that were most prevalent in each of the databases. (Figs. 6A-D).

\section{Correlation between NSUN6 expression levels and immune checkpoints and immune neoantigens in pan-cancer}

Immune checkpoints are a series of molecules that are expressed in immune cells and can modulate immune activity [24]. On the contrary, new tumor antigens are newborn antigens encoded by mutant genes in tumor cells. The immune activity of new tumor antigen is used to promote the synthesis of new antigen vaccine [25]. NSUN6's role in regulating the immune system was explored this time by examining the association between NSUN6 and immunological checkpoints and neoantigens. More than 40 checkpoints, including KICH, UVM, and NSUN6, were found to connect with NSUN6 expression levels in diverse cancers (Fig. 7A). In some cancers, NSUN6 was found to have an important function in modifying immunological checkpoints and may be able to boost immunity. In addition, we counted the amount of neoantigens present in each type of tumor in the current study. The results showed that NSUN6 was only correlated in BRCA $(R=-0.128, P=0.00055)$, THCA $(R=-0.013, P=0.0464)$ and STAD $(R=0.174, P=$ 0.0069) (Fig. 7B).

\section{Correlation between NSUN6 and tumor immune infiltration and the tumor microenvironment in pan-cancer}


The difference in the degree of infiltration of different immune cells is closely related to the progression and prognosis of the tumor[26]. By contrast, the tumor microenvironment consists of a variety of cells, ECM, and related factors that have an important impact on the diagnosis, survival, and clinical treatment sensitivity of the tumor[27]. As part of this work, we looked examined NSUN6's immune infiltration and the tumor microenvironment in various malignancies. A total of 3 most relevant tumors (BLCA, KIRC, KIRP) were identified after analyzing 33 tumors in the TCGA database. Among these tumors, NSUN6 was positively correlated with $B$ cells $(R=0.165, P<0.001)$, but negatively associated with $C D 4+T$ cells $(R=$ $-0.286, P<0.001), C D 8+T$ cells $(R=-0.376, P<0.001)$, Dendritic $(R=-0.506, P<0.001)$, Macrophages $(R=$ $-0.228, P<0.001)$, and Neutrophil cells $(R=-0.453, P<0.001)$ in BLCA. In addition, NSUN6 was positively correlated with CD4 $+T$ cells $(R=0.323, P<0.001)$, CD8 $+T$ cells $(R=0.139, P<0.001)$, Neutrophils $(R=$ $0.289, P<0.001)$, macrophages $(R=0.127, P<0.05)$ and dendritic cells $(R=0.113, P<0.001)$ in $K I R C$. Furthermore, NSUN6 was positively correlated with $C D 4+T$ cells $(R=0.144, P<0.05)$, Neutrophil cells $(R=$ $0.205, P<0.001)$, but negatively related to $B$ cells $(R=-0.199, P<0.001), C D 8+T$ cells $(R=-0.307, P<0.001)$, Macrophages $(\mathrm{R}=-0.219, \mathrm{P}<0.001)$ in $\mathrm{KIRP}($ Fig. 8A).

Researchers in this study looked at the link between NSUN6 and tumor immune infiltration as well as the tumor microenvironment in pan-cancer. Each tumor sample was evaluated using the $\mathrm{R}$ package (ESTIMATE) and the association between NSUN6 expression levels and the immunological score, stromal score, and the immune score of ESTIMATE was analyzed individually. BLCA $(R=-0.44, p<0.001)$, LGG $(R=-0.441, p<0.001), \operatorname{PRAD}(R=-0.31, p<0.001)$ stromal scores were negatively correlated with NSUN6 expression levels. We showed the top three tumors in which BLCA $(R=-0.44, p<0.001), G B M(R$ $=-0.432, p<0.001)$, THCA $(R=-0.23, p<0.001)$ immune scores were negatively correlated with NSUN6 expression levels in 33 tumors. In addition, the immune scores of $B L C A(R=-0.44, P<0.001), L G G G(R=-$ $0.441, P<0.001)$, GBM $(R=-0.432, P<0.001)$ ESTIMATE were found to be negatively correlated with NSUN6 expression levels (Fig. 8B). NSUN6 was found to be negatively related correlation with both tumor immune scores based on these findings.

\section{TMB and MSI analyses of NSUN6 expression in pan-cancer}

TMB can be used to assess the total number of substitutions and insertions/deletions per megabyte of exons in tumor samples and to predict the success and prognosis of immune-checkpoint therapy [28]. MSI is caused by a mismatch repair (MMR) gene defect and is associated with tumorigenesis [29]. We investigated the link between NSUN6 in TMB and MSI in the current study. A Spearman correlation analysis showed that NSUN6 expression levels were positively correlated with SKCM and STAD, and negatively with UCEC, UVM and BLCA, as well as TGCT, SARC, KIRC, KIRP, LGG and LIHC. This was in contrast to the correlations found between NSUN6 expression levels and SKCM, STAD, SARC, TGCT, DLBC and GBM (Fig. 9A). MSI cancers had NSUN6 expression levels identified using Spearman test analysis. According to Spearman test analysis, NSUN6 expression levels were positively connected with LUSC, LUAD, LGG, KIRP, HNSC, and GBM, ESCA, COAD, CHOL, UCEC, and THCA; negatively correlated with SKCM and TGCT; and unrelated to DLBC and LIHC (Fig. 9B). 


\section{In pan carcinoma, the expression of NSUN6 and MMR genes and methyl-transferases are linked}

This study used the TCGA database to examine the association between the NSUN6 gene and the MMR gene family (MLH1, MSH2, MSH6, PMS2, EPCAM). All 33 cancers except LIHC and UCS had NSUN6 expression levels that were associated with MMR genes, according to the study (Fig. 10A). NSUN6 expression levels were also shown to be strongly and positively linked with the expression levels of both methyltransferases (Fig. 10B). According to these findings, NSUN6 is involved in cancer development and has epigenetic features that govern a variety of malignancies.

\section{Discussion}

RNA methylation is a new frontier of research on epitopes, representing a new level of genetic information control[13]. Messenger RNA (mRNA), transporter RNA (tRNA) and enhancer RNA (enhancer RNA) have been proved to have many methylation markers, such as 5- methylcytosine ( $\mathrm{m} 5 \mathrm{c}), \mathrm{n}$-6- methyladenosine (m6A)[13]. Previous study demonstrated that RNA methylation is also associated with malignant tumors[30]. For example, an m6A-labeled eraser ALKBH5 has been shown to maintain the stemness of glioma[31].

Epigenetic marker, 5- methylcytosine gene $(5 \mathrm{mC})$, is stored on both DNA and RNA. Although the information of $5 \mathrm{mc}$ DNA methyltransferases (DNMTs) is available, some studies have shown that $5 \mathrm{mc}$ is deposited on RNA by a group of RNA methyltransferases called NSUNs[13]. These enzymes belong to Nol1P/Nop2/SUN domain[32], which including NSUN1, NSUN2, NSUN3, NSUN4, NSUN5, nsun6 and NSUN7[13].

NSUN6 (NOP2/Sun RNA Methyltransferase 6) is a Protein Coding gene, is involved in the regulation of signaling pathways including tumor proliferation, occurrence, and metastasis[33]. NSUN6, as a new m5C RNA methylation regulators, had the potential to become ideal diagnostic biomarker. Here, we discovered that nsun6 expression levels differed widely across a range of malignancies, and that these differences were directly linked to the prognosis of cancer patients. Furthermore, nsun6 overexpression has been linked to a poor prognosis in other studies. NSUN6 is also strongly linked to tumor immunity and interacts with a variety of tumor-specific immune cells. DNA methylation and ICP, TMB, MSI, MMRs, and MSI were all associated positively with NSUN6 expression. It is clear that NSUN6 is an independent biomarker for a wide range of malignancies, as evidenced by the results of this study. The extracellular matrix receptor interaction, metabolism, and cell adhesion in triple-negative breast cancer were all found to be influenced by NSUN6 deregulation (TNBC)[33]. C Cell-cell interaction and adhesion are critical mediators of cancer development, as they enhance cancer features such as immune evasion and metastatic spread, hence altering cancer prognosis [34]. These connections may account for the positive link between increased NSUN6 expression and poor prognosis in tumor patients, implying that NSUN6 can be employed as a prognostic biomarker for a variety of tumor types. 
There are tumor stem cells, immune cells and other molecules in the tumor microenvironment that are helpful to the occurrence and development of tumors[35].Generally, immunization of cells in tumor microenvironment (TME) is an integral part of an anti-tumor strategy to prevent tumor cells from being killed[36]. There is a scarcity of studies on the interaction of NSUN6 with immune cells or the immunological microenvironment. The purpose of this study was to determine the relationship between NSUN6 expression and immune cells. We discovered a substantial association between NSUN6 expression and immune cell infiltration levels in a variety of cancers, most notably BLCA, KIRC, and KIRP. These findings suggested that NSUN6 was involved in the recruitment and control of immune infiltrate cells in a variety of malignancies.

Immune-checkpoint inhibitors have been proved to play an important role in tumor treatment[37, 38]. In addition, the application of immune checkpoint inhibitor enhanced cancer immunity via blocking immune checkpoint receptors, inhibiting tumor metastasis and recurrence, and reducing off-target adverse reactions[39]. nhibition of the PD-1/pd-11 axis with immune checkpoint inhibitors (ICI) such as nivolumab and pembrolizumab has emerged as a new therapy option for metastatic gastric cancer [40]. Previous studies have shown that immune checkpoint blockers have anti-tumor drug resistance[41]. Our research discovered a significant correlation between NSUN6 and many immune checkpoint genes in a variety of tumor types, implying that aberrant NSUN6 expression may be associated with chemotherapy treatment resistance. As a result, NSUN6 may be employed as a novel anticancer immunotherapy target or in combination with established checkpoint inhibitors to increase tumor immunity and response. Additionally, the findings indicate that NSUN6 may be a suitable target for anti-tumor immunotherapy. Combining the deletion of the NSUN6 gene with chemotherapeutic medicines may become a novel antitumor technique.

A major role of the match repair is to identify errors in the DNA replication process and repair them in order to maintain genetic stability[42]. Mismatch repair (MMR) proteins cooperate with each other to identify, delete and repair mismatched bases. Mismatch repair function can lead to errors in DNA replication, resulting in abnormal DNA structure, the emergence of highly mutated subtypes and the emergence of tumors $[43,44]$. For instance, mutations in MMR genes that result in the occurrence of colorectal cancer[45]. To assess tumor somatic mutations, we examined the association between the expression of five MMR-related genes, including MLH1, MSH2, MSH6, PMS2, EPCAM, and NSUN6.

DNA methylation is one of the most studied epigenetic modifications in mammals[46].

Additionally, DNA methyltransferase (DNMT), the primary regulator of DNA methylation, is comprised of the enzymes DNMT1, DNMT2, DNMT3a, and DNMT3b $[47,48]$. Aberrant DNA methylation occurs at every stage of tumor occurrence and development, and DNMT is strongly expressed for the first time in precancerous lesions, implying that abnormal DNA methylation may be one of the early molecular events associated with tumor occurrence and development $[49,50]$. Furthermore, DNMT was found to be strongly linked with tumor patient prognosis and may be employed as a target for tumor therapy [51-54]. The data indicated a strong correlation between NSUN6 expression and MMRs and DNA methylation, 
particularly in COAD, HNSC, STAD, LAML, UCEC, and READ. As a result, it is plausible to conclude that NSUN6 plays a critical role in mismatch repair and DNA methylation in pan-cancer, and that additional research on NSUN6 expression and tumor immunity may aid in the development of novel immunotherapy strategies.

Our research, however, has certain drawbacks. To begin, it is suggested that the degree of NSUN6 expression is associated with the prognosis and immune infiltration of universal cancer. At the moment, there is no direct evidence that NSUN6 influences cancer prognosis via immunological checkpoints or tumor alterations. Second, assessment and analysis are carried out by merging numerous information databases, however the majority of data in the database originates from gene chip and sequencing experiments, leaving out sufficient experimental and clinical data. This will have some effect on the results, and so in vitro and in vivo research are required to confirm and clarify the correlation between NSUN6 expression and tumor immune infiltration. Finally, the expression of the NSUN6 gene is determined using mRNA levels from the aforementioned databases, which may not adequately reflect the level of functional proteins.

\section{Conclusion}

We discovered that NSUN6 was variably expressed in pan-cancers and that aberrant expression was related with tumor progression in the current investigation. Immune checkpoints, immune cell infiltration, the tumor microenvironment, TMB, MSI, and DNA methylation were all related with abnormal NSUN6 expression. NSUN6 has potential as a tumor prognostic marker.

\section{Abbreviations In Tcga}

Adrenocortical carcinoma ACC; Bladder urothelial carcinoma BLCA; Breast invasive carcinoma BRCA; Cervical squamous cell carcinoma and endocervical adenocarcinoma CESC; Cholangiocarcinoma $\mathrm{CHOL}$; Colon adenocarcinoma COAD; Rectum adenocarcinoma READ; Lymphoid neoplasm diffuse large B-cell lymphoma DLBC; Esophageal carcinoma ESCA; Glioblastoma multiforme GBM; Head and neck squamous cell carcinoma HNSC; Kidney chromophobe KICH; Kidney renal clear cell carcinoma KIRC; Kidney renal papillary cell carcinoma KIRP; Acute myeloid leukemia LAML; Low grade glioma LGG; Liver hepatocellular carcinoma LIHC; Lung adenocarcinoma LUAD; Lung squamous cell carcinoma LUSC; Mesothelioma MESO; Ovarian serous cystadenocarcinoma OV; Pancreatic adenocarcinoma PAAD; Pheochromocytoma and paraganglioma PCPG; Prostate adenocarcinoma PRAD; Rectum adenocarcinoma READ; Sarcoma SARC; Skin cutaneous melanoma SKCM; Stomach adenocarcinoma STAD; Stomach and esophageal carcinoma STES; Testicular germ cell tumors TGCT; Thyroid carcinoma THCA; Thymoma THYM; Uterine corpus endometrial carcinoma UCEC; Uveal melanoma UVM;

\section{Declarations}

\section{Data availability statement}


This study investigated publicly available datasets. This data can be found here: https://portal.gdc.cancer.gov/, https://gtexportal.org/, https://portals.broadinstitute.org/, https:// cistrome.shinyapps.io/timer/.

\section{Author contributions}

The entire process, from software development to formal analysis, original draft writing, visualization, and supervision, was conducted mostly by Baishun Ma. Pu Wang also contributes to the supervision of this article's work. The manuscript was reviewed by all authors. The final manuscript was read and approved by all writers.

\section{Acknowledgement}

The authors would like acknowledge Hangzhou Mugu science and technology Co., Ltd. for developing the sangerbox tool. Grammar correction of this article by Quillbot (https://quillbot.com/). The authors like to express their gratitude for their assistance with this work.

\section{References}

1. Budnik, L.T., et al., Pollution in living and working environments, climate variability, and their impact on non-communicable disease burden. Sci Total Environ, 2019. 660: p. 593-594.

2. Jemal, A., et al., Global patterns of cancer incidence and mortality rates and trends. Cancer Epidemiol Biomarkers Prev, 2010. 19(8): p. 1893-907.

3. Seymour, L., et al., iRECIST: guidelines for response criteria for use in trials testing immunotherapeutics. Lancet Oncol, 2017. 18(3): p. e143-e152.

4. Straker, N., The Treatment of Cancer Patients Who Die. Psychodyn Psychiatry, 2020. 48(1): p. 1-25.

5. Malik, D., et al., Futuristic approach to cancer treatment. Gene, 2021. 805: p. 145906.

6. Lin, Y.M., et al., Tumor Biomarkers and Interventional Oncology: Impact on Local Outcomes for Liver and Lung Malignancy. Curr Oncol Rep, 2021. 23(6): p. 67.

7. Nagy, Á., G. Munkácsy, and B. Győrffy, Pancancer survival analysis of cancer hallmark genes. Sci Rep, 2021. 11(1): p. 6047.

8. Reid, R., P.J. Greene, and D.V. Santi, Exposition of a family of RNA $m(5) C$ methyltransferases from searching genomic and proteomic sequences. Nucleic Acids Res, 1999. 27(15): p. 3138-45.

9. Blanco, S. and M. Frye, Role of RNA methyltransferases in tissue renewal and pathology. Curr Opin Cell Biol, 2014. 31: p. 1-7.

10. Alshaker, H., et al., Transcriptome-Wide Effects of Sphingosine Kinases Knockdown in Metastatic Prostate and Breast Cancer Cells: Implications for Therapeutic Targeting. Front Pharmacol, 2019. 10: p. 303.

11. Zheng, X., et al., The sphingosine kinase-1/sphingosine-1-phosphate axis in cancer: Potential target for anticancer therapy. Pharmacol Ther, 2019. 195: p. 85-99. 
12. Okamoto, M., et al., tRNA modifying enzymes, NSUN2 and METTL 1, determine sensitivity to 5fluorouracil in HeLa cells. PLoS Genet, 2014. 10(9): p. e1004639.

13. Awah, C.U., et al., NSUN6, an RNA methyltransferase of 5-mC controls glioblastoma response to temozolomide (TMZ) via NELFB and RPS6KB2 interaction. Cancer Biol Ther, 2021. 22(10-12): p. 587597.

14. Xue, M., et al., Gene signatures of $m 5 C$ regulators may predict prognoses of patients with head and neck squamous cell carcinoma. Am J Transl Res, 2020. 12(10): p. 6841-6852.

15. Zhang, Z., X. Zhang, and A. Huang, Aggresome-Autophagy Associated Gene HDAC6 Is a Potential Biomarker in Pan-Cancer, Especially in Colon Adenocarcinoma. Front Oncol, 2021. 11: p. 718589.

16. Tomczak, K., P. Czerwińska, and M. Wiznerowicz, The Cancer Genome Atlas (TCGA): an immeasurable source of knowledge. Contemp Oncol (Pozn), 2015. 19(1a): p. A68-77.

17. The Genotype-Tissue Expression (GTEx) project. Nat Genet, 2013. 45(6): p. 580-5.

18. Barretina, J., et al., The Cancer Cell Line Encyclopedia enables predictive modelling of anticancer drug sensitivity. Nature, 2012. 483(7391): p. 603-7.

19. Li, T., et al., TIMER: A Web Server for Comprehensive Analysis of Tumor-Infiltrating Immune Cells. Cancer Res, 2017. 77(21): p. e108-e110.

20. Robinson, M.D., D.J. McCarthy, and G.K. Smyth, edgeR: a Bioconductor package for differential expression analysis of digital gene expression data. Bioinformatics, 2010. 26(1): p. 139-40.

21. Ito, K. and D. Murphy, Application of ggplot2 to Pharmacometric Graphics. CPT Pharmacometrics Syst Pharmacol, 2013. 2(10): p. e79.

22. Subramanian, A., et al., Gene set enrichment analysis: a knowledge-based approach for interpreting genome-wide expression profiles. Proc Natl Acad Sci U S A, 2005. 102(43): p. 15545-50.

23. Yu, G., et al., clusterProfiler: an R package for comparing biological themes among gene clusters. Omics, 2012. 16(5): p. 284-7.

24. Zhou, B. and S. Gao, Pan-Cancer Analysis of FURIN as a Potential Prognostic and Immunological Biomarker. Front Mol Biosci, 2021. 8: p. 648402.

25. Cerezo-Wallis, D. and M.S. Soengas, Understanding Tumor-Antigen Presentation in the New Era of Cancer Immunotherapy. Curr Pharm Des, 2016. 22(41): p. 6234-6250.

26. Sokratous, G., S. Polyzoidis, and K. Ashkan, Immune infiltration of tumor microenvironment following immunotherapy for glioblastoma multiforme. Hum Vaccin Immunother, 2017. 13(11): p. 2575-2582.

27. Gajewski, T.F., H. Schreiber, and Y.X. Fu, Innate and adaptive immune cells in the tumor microenvironment. Nat Immunol, 2013. 14(10): p. 1014-22.

28. Merino, D.M., et al., Establishing guidelines to harmonize tumor mutational burden (TMB): in silico assessment of variation in TMB quantification across diagnostic platforms: phase I of the Friends of Cancer Research TMB Harmonization Project. J Immunother Cancer, 2020. 8(1).

29. Schrock, A.B., et al., Tumor mutational burden is predictive of response to immune checkpoint inhibitors in MSI-high metastatic colorectal cancer. Ann Oncol, 2019. 30(7): p. 1096-1103. 
30. Lesbirel, S. and S.A. Wilson, The m(6)A-methylase complex and mRNA export. Biochim Biophys Acta Gene Regul Mech, 2019. 1862(3): p. 319-328.

31. Zhang, S., et al., m(6)A Demethylase ALKBH5 Maintains Tumorigenicity of Glioblastoma Stem-like Cells by Sustaining FOXM1 Expression and Cell Proliferation Program. Cancer Cell, 2017. 31(4): p. 591-606.e6.

32. Haag, S., et al., NSUN6 is a human RNA methyltransferase that catalyzes formation of m5C72 in specific tRNAs. Rna, 2015. 21(9): p. 1532-43.

33. Huang, Z., et al., Prognostic Significance and Tumor Immune Microenvironment Heterogenicity of m5C RNA Methylation Regulators in Triple-Negative Breast Cancer. Front Cell Dev Biol, 2021. 9: p. 657547.

34. Läubli, H. and L. Borsig, Altered Cell Adhesion and Glycosylation Promote Cancer Immune Suppression and Metastasis. Front Immunol, 2019. 10: p. 2120.

35. Arneth, B., Tumor Microenvironment. Medicina (Kaunas), 2019. 56(1).

36. Jarosz-Biej, M., et al., Tumor Microenvironment as A "Game Changer" in Cancer Radiotherapy. Int J Mol Sci, 2019. 20(13).

37. Doroshow, D.B., et al., PD-L1 as a biomarker of response to immune-checkpoint inhibitors. Nat Rev Clin Oncol, 2021. 18(6): p. 345-362.

38. Carlino, M.S., J. Larkin, and G.V. Long, Immune checkpoint inhibitors in melanoma. Lancet, 2021. 398(10304): p. 1002-1014.

39. Bagchi, S., R. Yuan, and E.G. Engleman, Immune Checkpoint Inhibitors for the Treatment of Cancer: Clinical Impact and Mechanisms of Response and Resistance. Annu Rev Pathol, 2021. 16: p. 223249.

40. Kono, K., S. Nakajima, and K. Mimura, Current status of immune checkpoint inhibitors for gastric cancer. Gastric Cancer, 2020. 23(4): p. 565-578.

41. Wang, Z. and X. Wu, Study and analysis of antitumor resistance mechanism of PD1/PD-L 1 immune checkpoint blocker. Cancer Med, 2020. 9(21): p. 8086-8121.

42. Fishel, R., Mismatch repair. J Biol Chem, 2015. 290(44): p. 26395-403.

43. Baretti, M. and D.T. Le, DNA mismatch repair in cancer. Pharmacol Ther, 2018. 189: p. 45-62.

44. Caja, F., et al., DNA Mismatch Repair Gene Variants in Sporadic Solid Cancers. Int J Mol Sci, 2020. 21(15).

45. De' Angelis, G.L., et al., Microsatellite instability in colorectal cancer. Acta Biomed, 2018. 89(9-s): p. 97-101.

46. Kulis, M. and M. Esteller, DNA methylation and cancer. Adv Genet, 2010. 70: p. 27-56.

47. Ma, F., Q. Zhang, and C.Y. Zhang, Nanomaterial-based biosensors for DNA methyltransferase assay. J Mater Chem B, 2020. 8(16): p. 3488-3501.

48. Poh, W.J., C.P. Wee, and Z. Gao, DNA Methyltransferase Activity Assays: Advances and Challenges. Theranostics, 2016. 6(3): p. 369-91. 
49. Etoh, T., et al., Increased DNA methyltransferase 1 (DNMT1) protein expression correlates significantly with poorer tumor differentiation and frequent DNA hypermethylation of multiple $C p G$ islands in gastric cancers. Am J Pathol, 2004. 164(2): p. 689-99.

50. Nosho, K., et al., DNMT3B expression might contribute to $C p G$ island methylator phenotype in colorectal cancer. Clin Cancer Res, 2009. 15(11): p. 3663-71.

51. Brueckner, B., D. Kuck, and F. Lyko, DNA methyltransferase inhibitors for cancer therapy. Cancer J, 2007. 13(1): p. 17-22.

52. Martisova, A., et al., DNA Methylation in Solid Tumors: Functions and Methods of Detection. Int J Mol Sci, 2021. 22(8).

53. Mahmoud, A.M. and M.M. Ali, Methyl Donor Micronutrients that Modify DNA Methylation and Cancer Outcome. Nutrients, 2019. 11(3).

54. Zafon, C., et al., DNA methylation in thyroid cancer. Endocr Relat Cancer, 2019. 26(7): p. R415-r439.

\section{Figures}

A

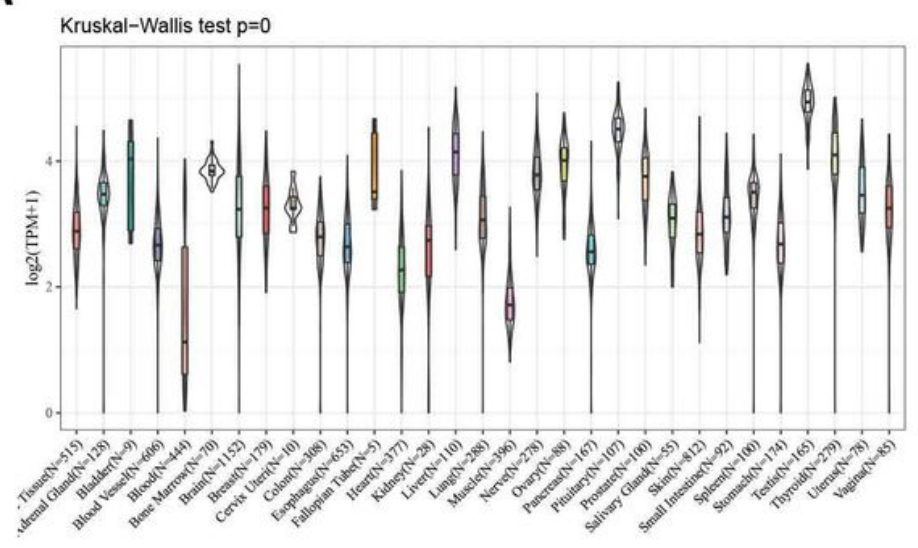

C

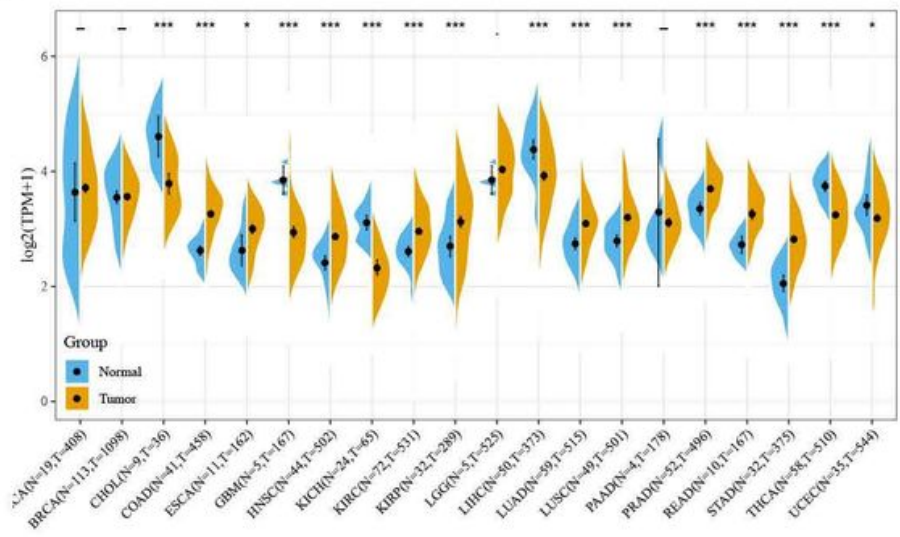

B

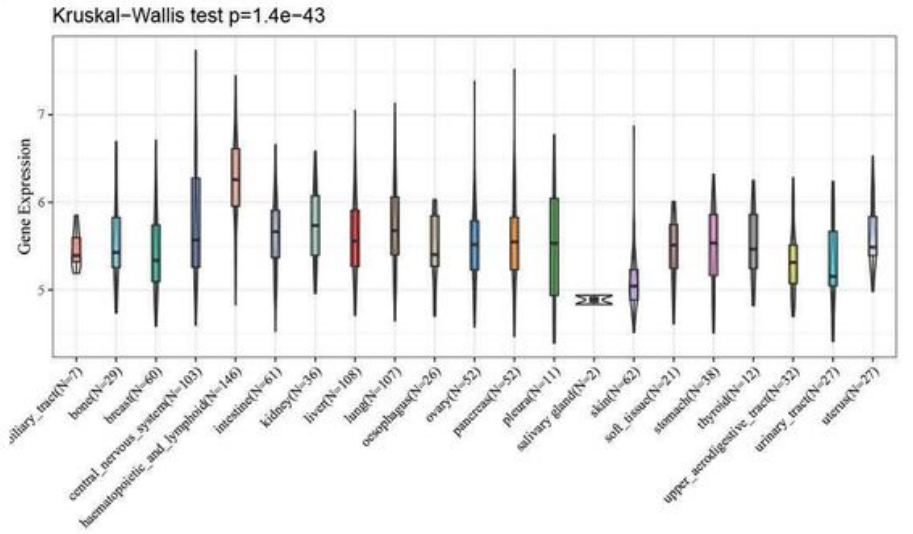

D

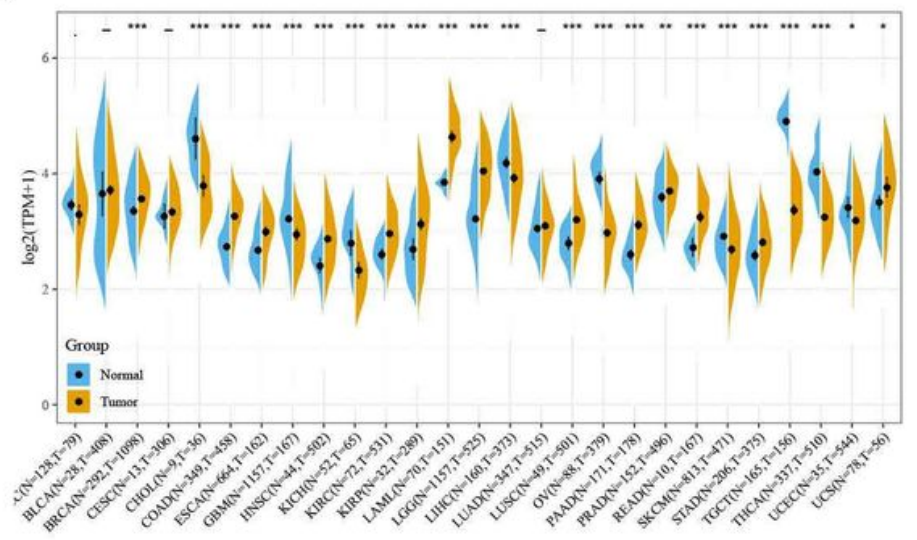

\section{Figure 1}

Levels of NSUN6 expression in pan-cancer. (A) Using the GTEx dataset, we analyzed NSUN6 expression levels in pan-cancer. (B) Using the CCLE dataset, analysis of NSUN6 expression levels in various cancer 
cells. (C) Using the TCGA database, analysis of NSUN6 expression levels in pan-cancer. (D) Integration of the GTEx and TCGA databases in order to obtain 27 tumor modules and analyze their NSUN6 expression levels. * indicates a P value of 0.05 in comparison to the control, ** indicates a $P$ value of 0.01 in comparison to the control, and $* \star \star$ indicates a $\mathrm{P}$ value of 0.001 in comparison to the control.

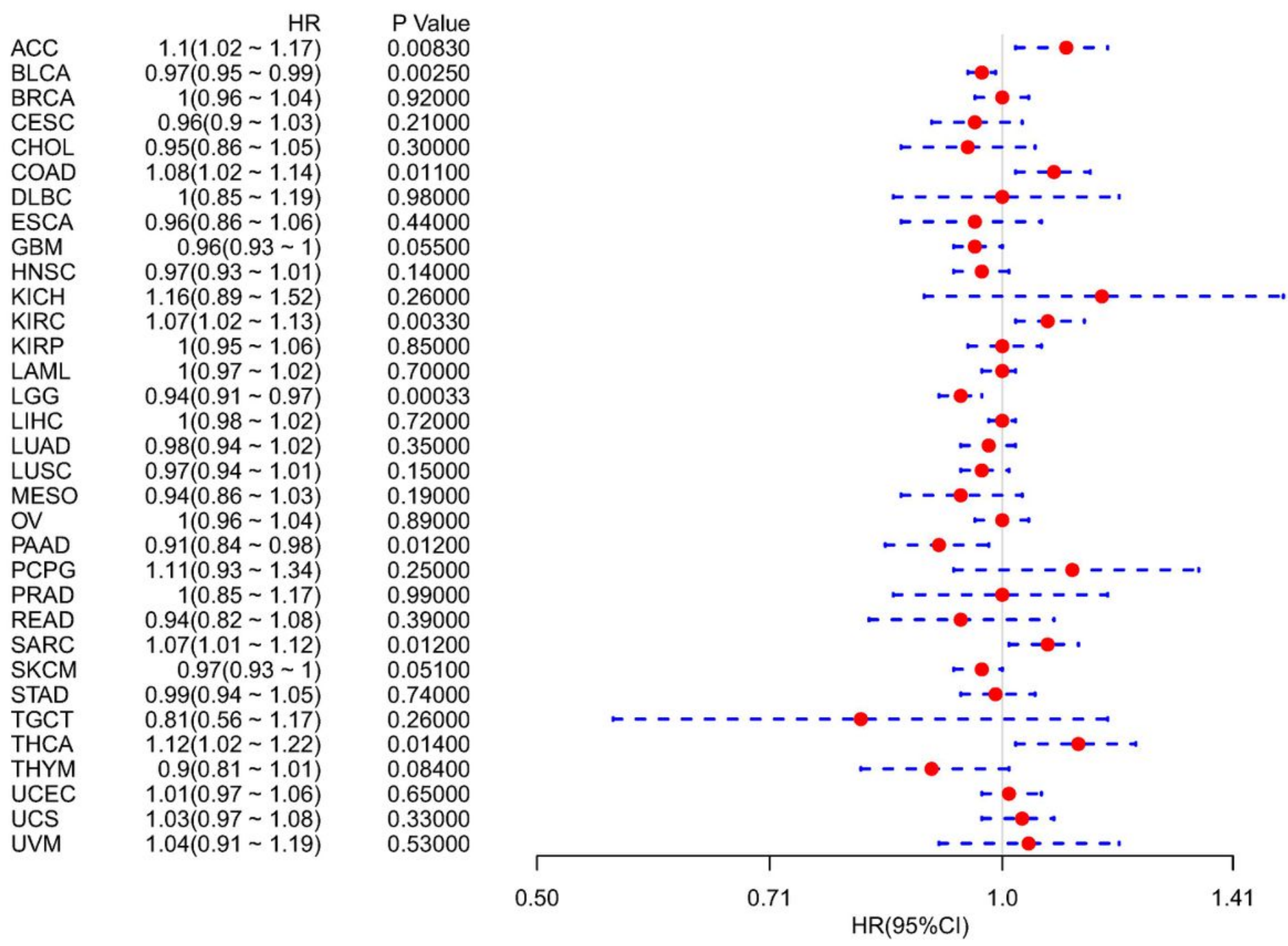

Figure 2

One-way Cox regression analysis of the relationship between NSUN6 and OS in 33 tumors, illustrated with forest plots. 

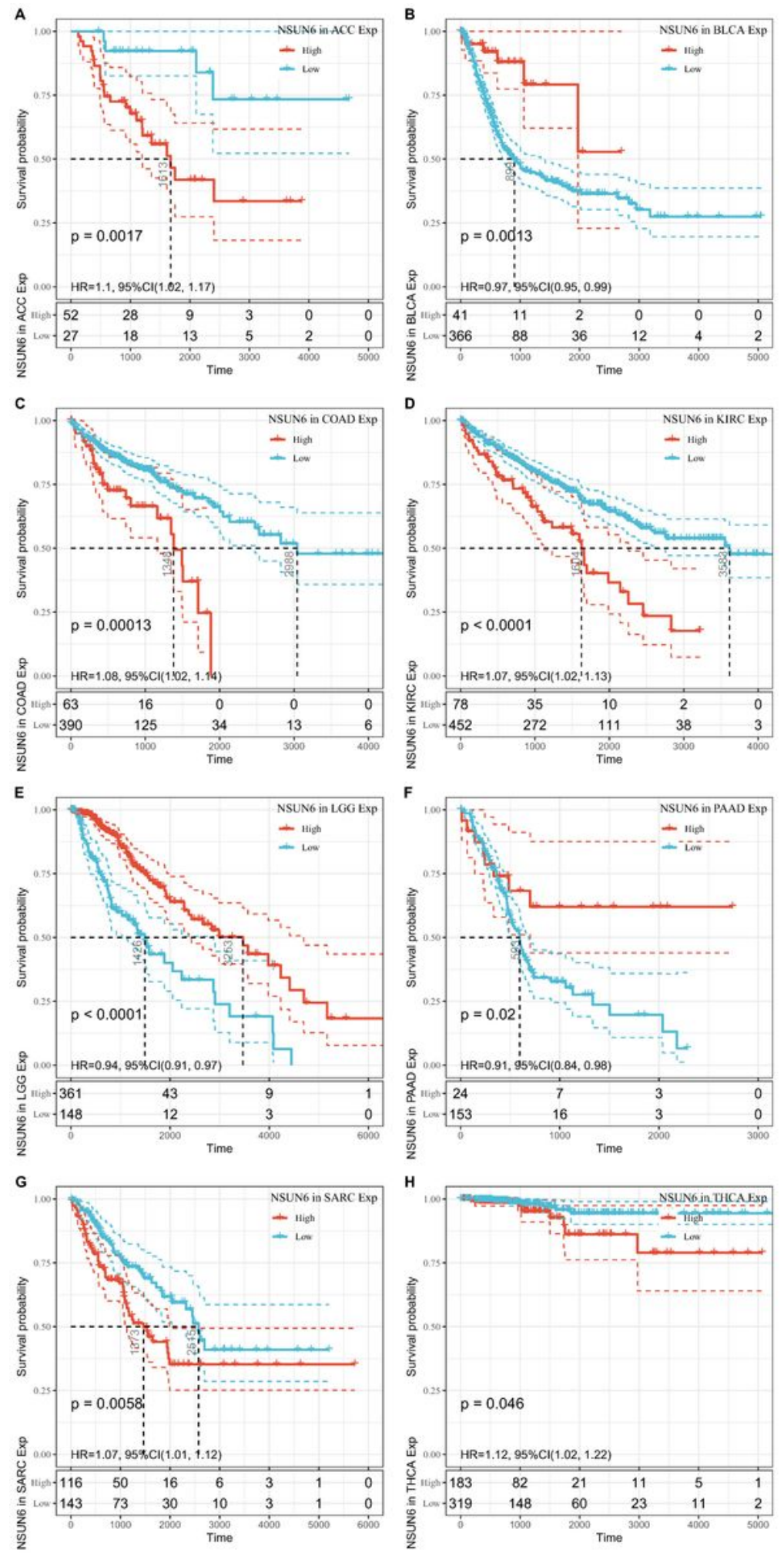

Figure 3

Relationship between NSUN6 and PAAD, THYM, and UVM OS. (A) The relationship between NSUN6 high and low expression levels and PAAD OS was analyzed using $\mathrm{K}-\mathrm{M}$ survival. (B) Relationship between NSUN6 high and low expression levels and THYM OS using K-M survival analysis. (C) The relationship between NSUN6 high and low expression levels and UVM OS using K-M survival analysis. 


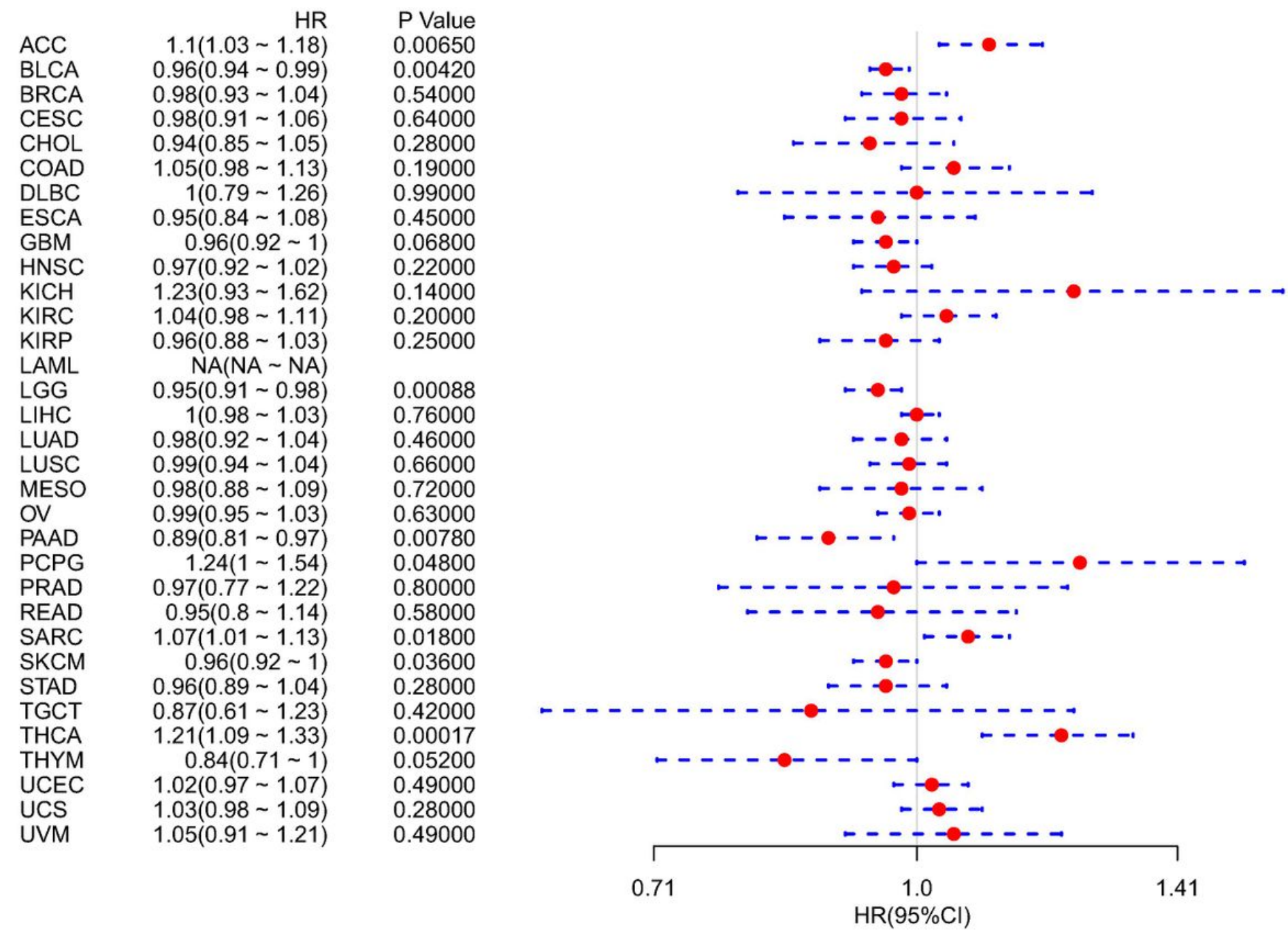

Figure 4

Analysis of the relationship between NSUN6 and DSS in 33 tumors using one-way Cox regression, presented using forest plots. 

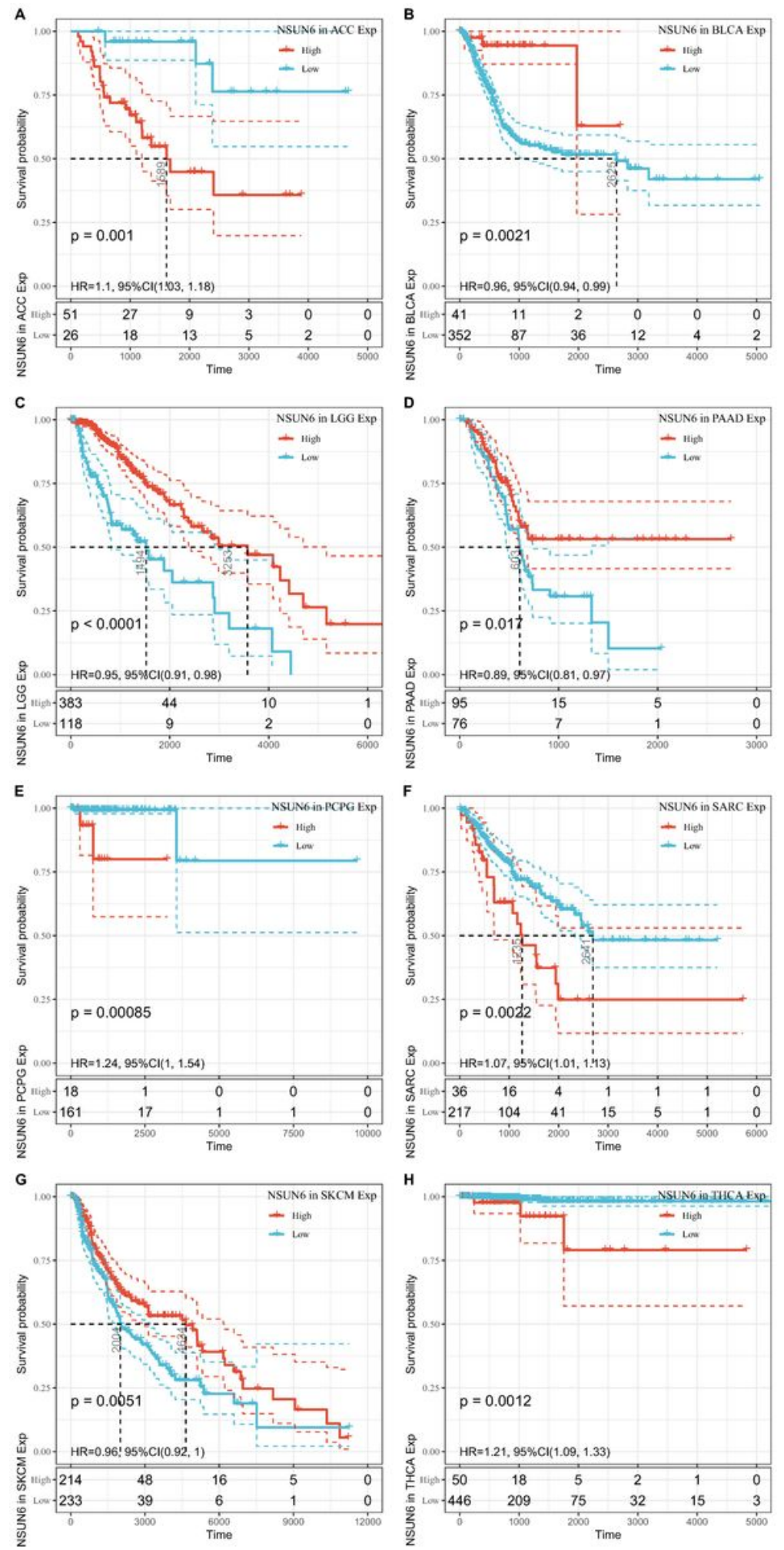

Figure 5

Relationship between NSUN6 and KIRP, THCA, THYM, UVM DSS. (A) The relationship between NSUN6 high and low expression levels and KIRP DSS was analyzed by K-M survival. (B) The relationship between NSUN6 high and low expression levels and THCA DSS was analyzed using K-M survival. (C) Relationship between NSUN6 high and low expression levels and THYM DSS using K-M survival 
analysis. (D) Relationship between NSUN6 high and low expression levels and UVM DSS using K-M survival analysis.

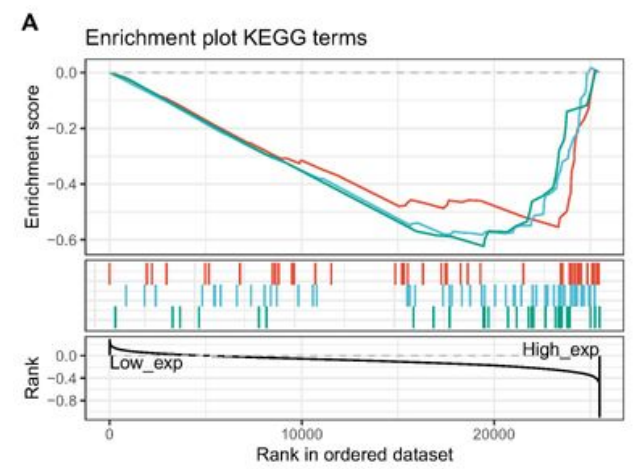

C

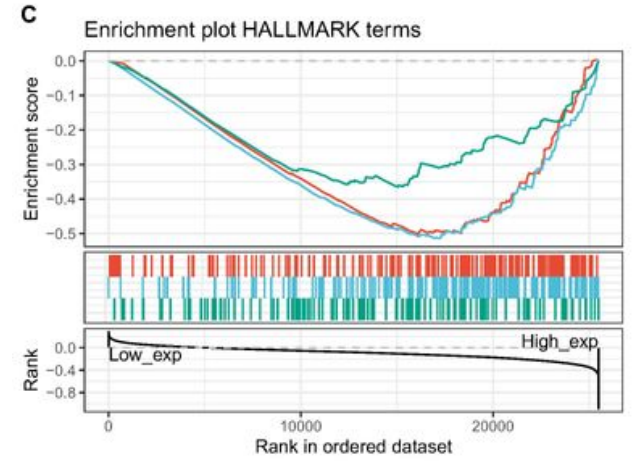

Term

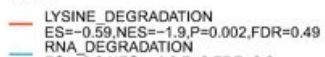

- RNA DEGRADATION $=0.002$, DDR $=0.09$

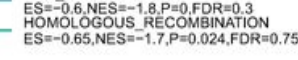

Term

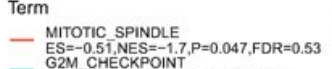

- ES $=-0.51, \mathrm{~N}$. NES $=-1.7, \mathrm{P}=0.047, \mathrm{FDR}=0$ — SPERMATOGENESS
B Enrichment plot KEGG terms

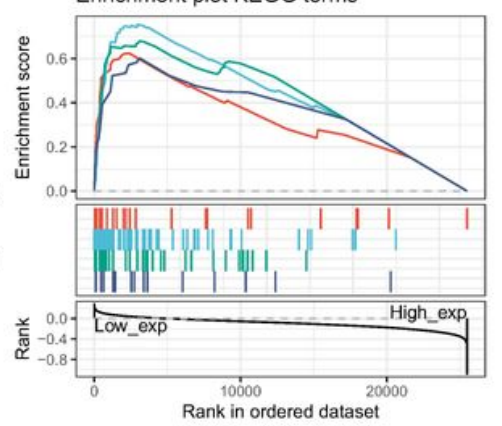

D

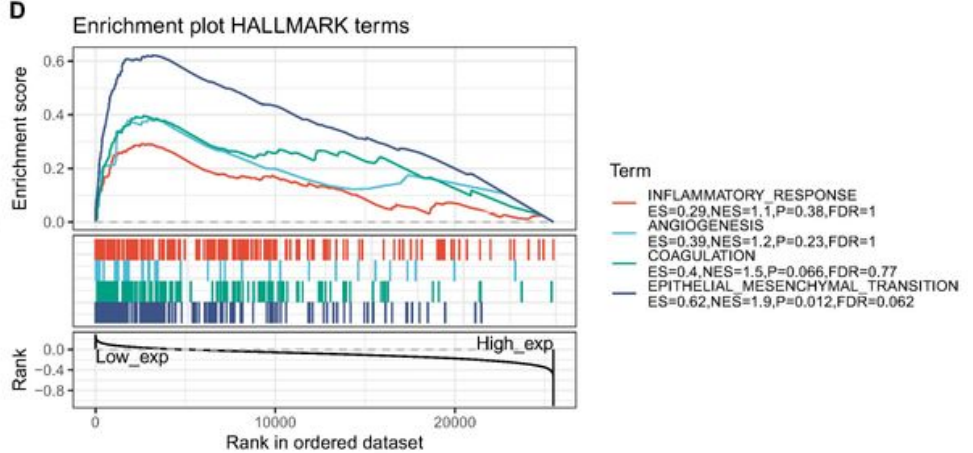

\section{Figure 6}

Enrichment of NSUN6 signaling in KEGG and markers. (A) and (B) GSEA analysis of the top three NSUN6signaling pathway correlations in the KEGG database. (C) and (D) GSEA analysis of NSUN6 using the marker dataset's top three signaling pathway correlations. 
A

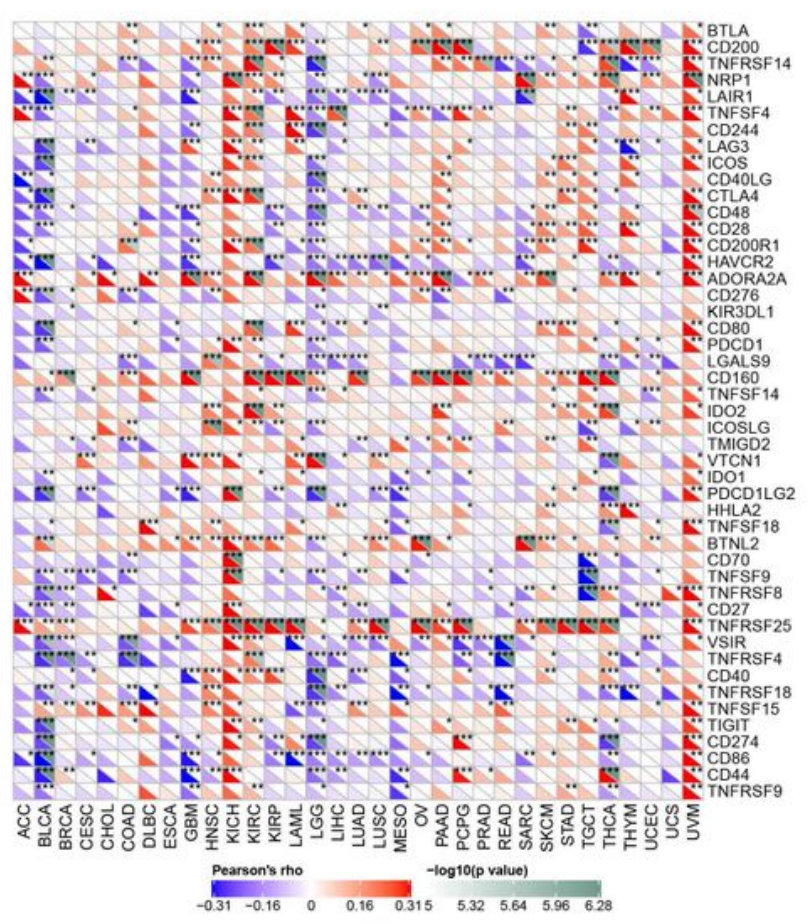

B
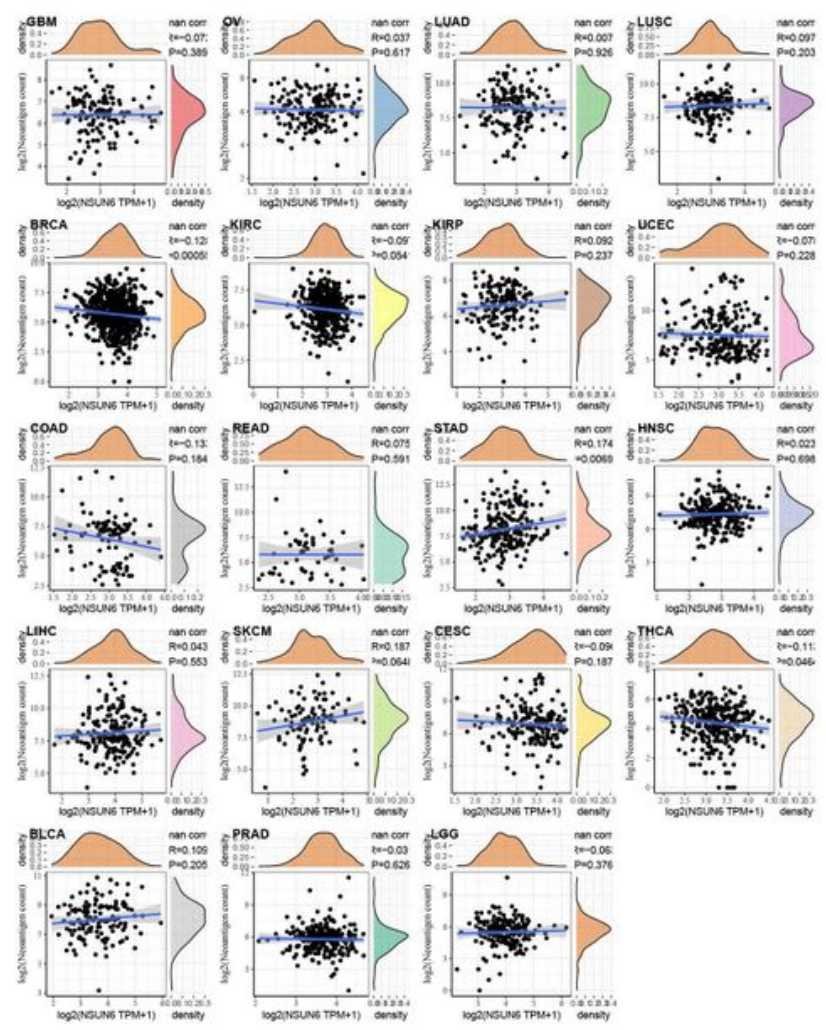

\section{Figure 7}

Analysis of the correlation between NSUN6 expression levels and immunological checkpoints and neoantigens. (A) Correlation analysis between the levels of NSUN6 expression and immune checkpoint gene expression in distinct malignancies. (B) Correlation analysis of NSUN6 expression levels in various tumors with the number of neoantigens in tumors. * indicates correlation difference of $P<0.05$, ** indicates correlation difference of $\mathrm{P}<0.01$, $* * *$ indicates correlation difference of $\mathrm{P}<0.001$ 
A
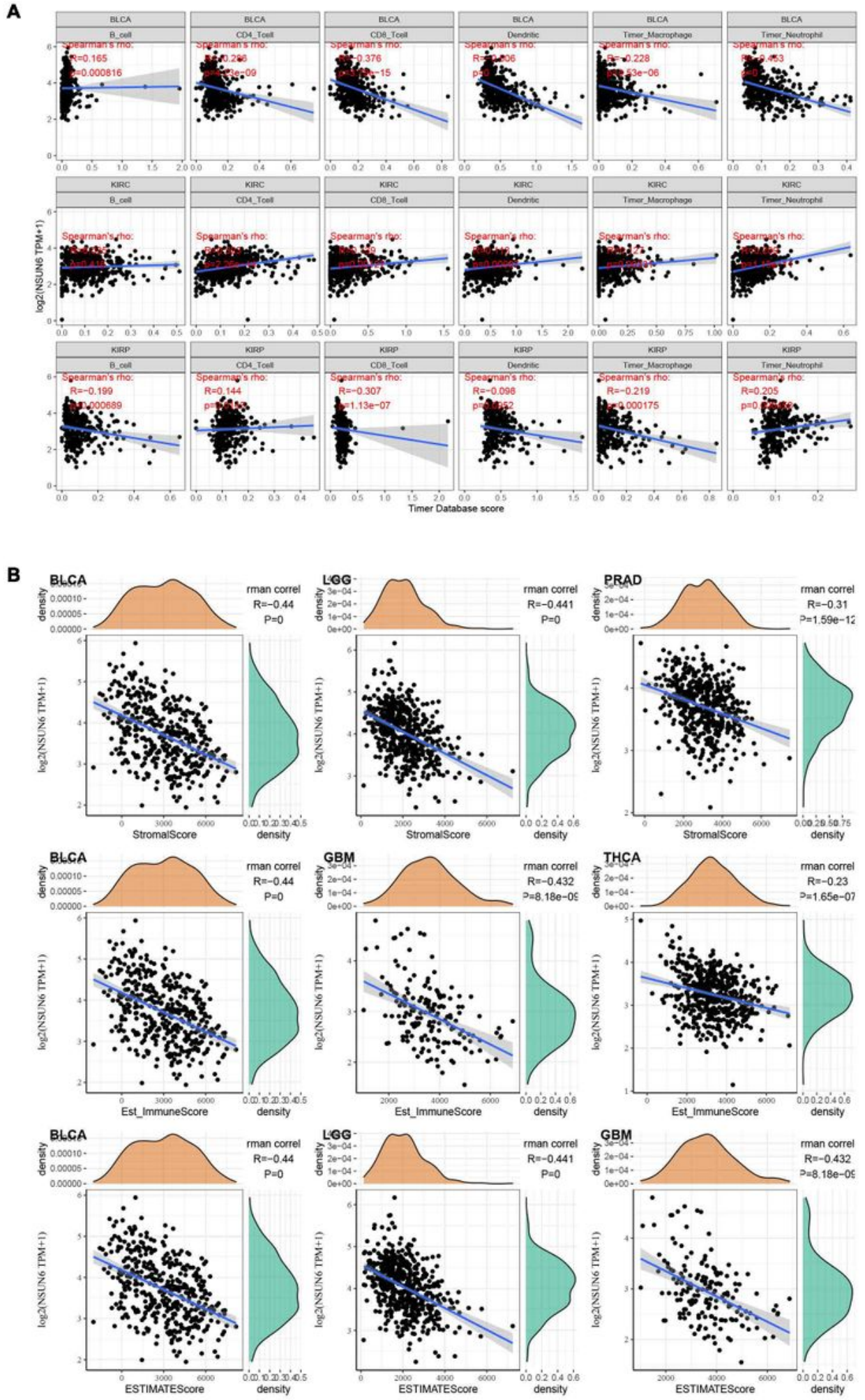

Figure 8

Correlation between NSUN6 expression and immune infiltration and microenvironment of tumors. (A) Analysis of the correlation between NSUN6 expression and immune cell infiltration in HNSC, KIRC, and PAAD. (B) Correlation between NSUN6 expression levels and ESTIMATES' immunological, stromal, and immune scores. 
A

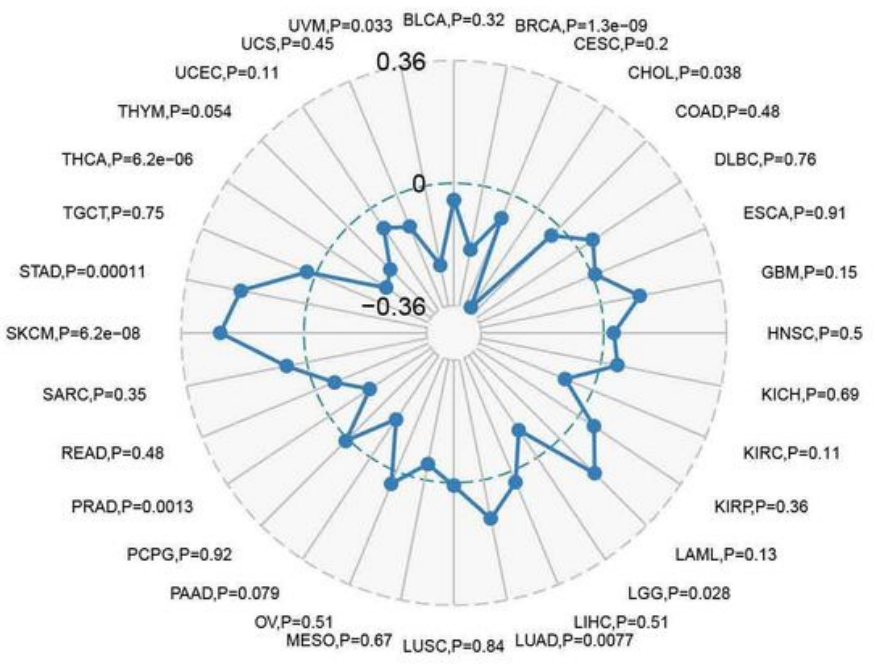

B

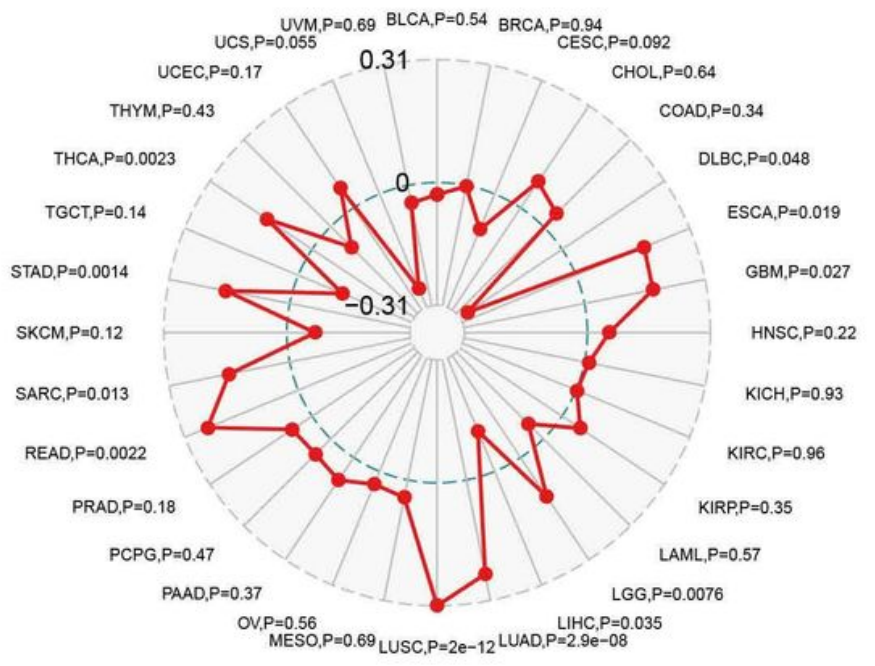

\section{Figure 9}

TMB and MSI analyses of the level of NSUN6 expression in relation to (A) Spearman correlation analysis was used to determine the association between NSUN6 and TMB in pan-cancer. (B) Spearman correlation analysis was used to determine the association between NSUN6 and MSI in pan-cancer.

A

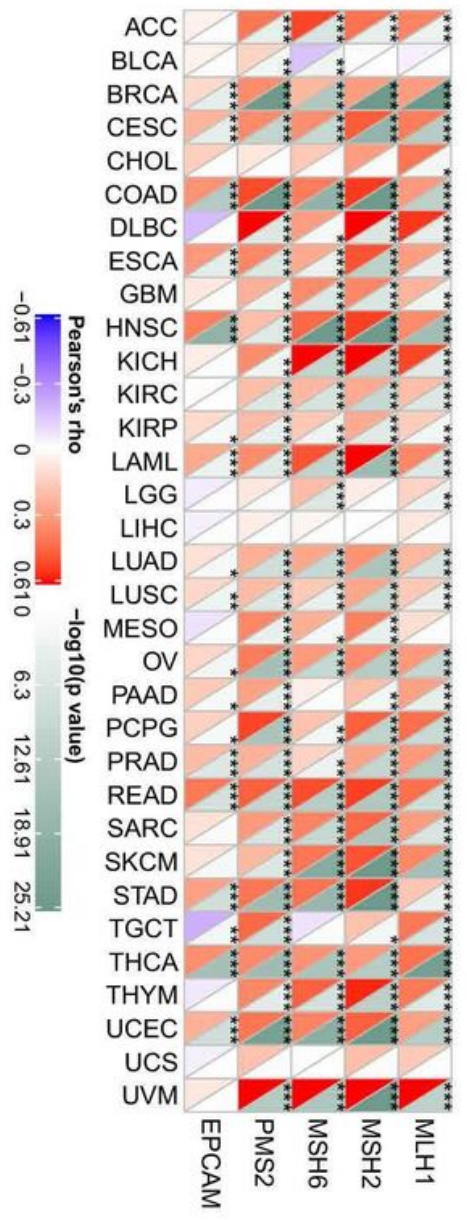

B

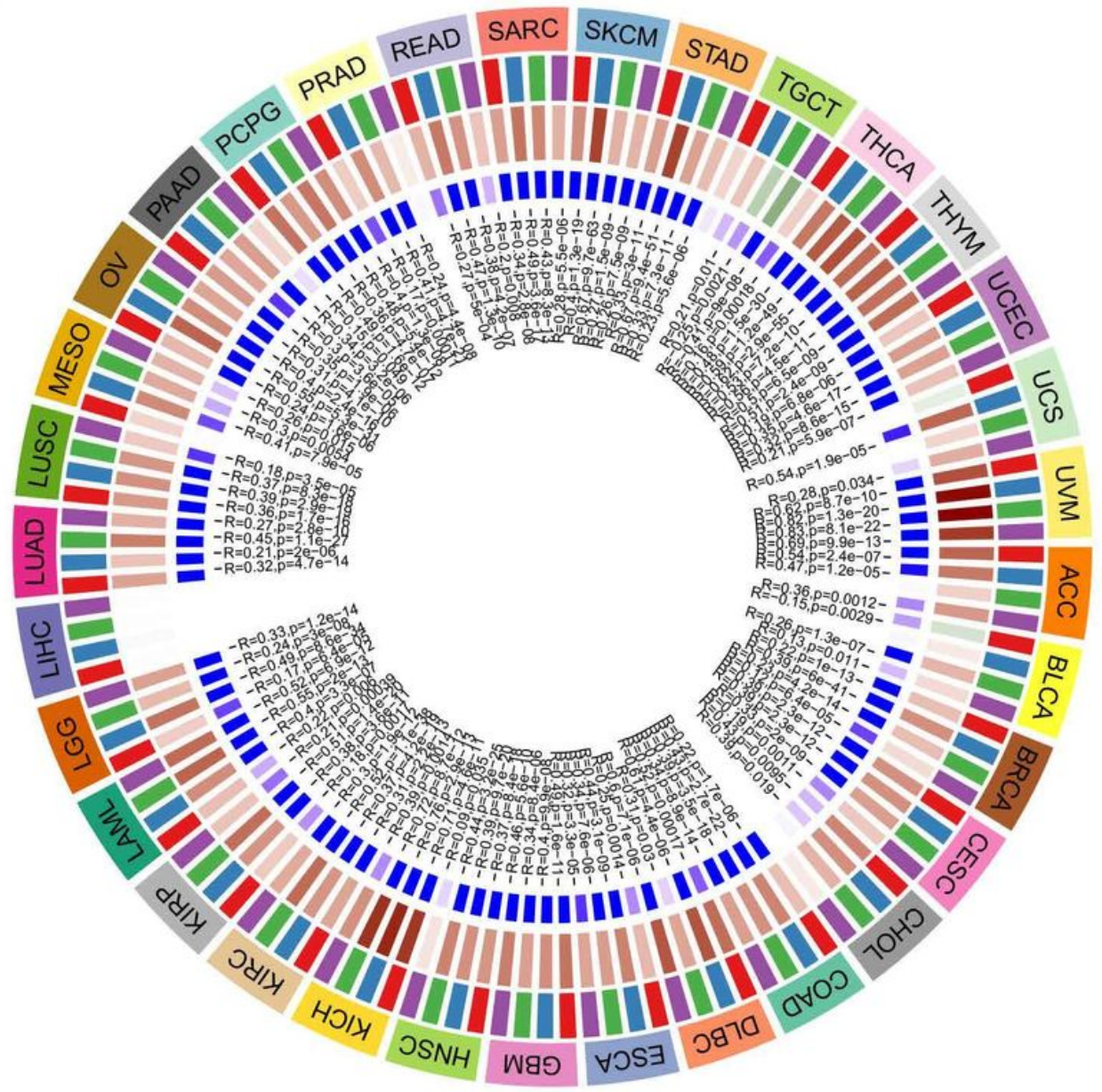


Figure 10

Correlation between the level of NSUN6 expression and the MMR gene and methyl transferase. (A) Spearman test to analyze the correlation between NSUN6 expression level and MMR gene in various tumors. (B) Spearman test to analyze the correlation between NSUN6 expression level and methyl transferase in various tumors. * Indicates that EPCAM, PMS2, MSH6, MSH2, MLH1 and NSUN6 are correlated in tumors, $\mathrm{P}<0.05$. ** Indicating that EPCAM, PMS2, MSH6, MSH2, MLH1 and NSUN6 are correlated in tumors, $\mathrm{P}<0.01$. ${ }^{* \star *}$ means EPCAM, PMS2, MSH6, MSH2, MLH1 and NSUN6 are correlated in tumors, $\mathrm{P}<0.001$. 